\title{
Everything is in The Vagus Nerve: What is The Relationship Between Chronic Fatigue Syndrome (CFS) and Coronavirus?
}

\section{Pr. Sakhri Selma*}

*Corresponding author: Pr. Sakhri Selma

Address: Medical oncology department - Algeria

e-mail $₫$ sakhri_selma@yahoo.fr

Received: 01 April 2020; Accepted: 07 April 2020

Definition

The vagus nerve, also known as the pneumogastric nerve, is the tenth pair of cranial nerves, involved in many functions of the body.

The sensory vagus nerve contains chemoreceptors sensitive to the presence of proinflammatory cytokines. It innervates the tissues that are often the first points of contact for foreign pathogens, such as the lining of the esophagus, the gastrointestinal lining, the lungs and lymph nodes. The vagus nerve also innervates most other important organs of the trunk such as the spleen, liver, heart, bladder, and pancreas.

\section{Chronic Fatigue Syndrome}

Chronic Fatigue Syndrome (CFS) is often a state of constant intense exhaustion that is not alleviated by rest or sleep. A diagnosis of CFS is given in the absence of alternative diagnostics. The prognosis is poor. The cause of CFS is unknown and the source of much controversy and debate. Previous studies on CFS patients have reported a variety of viral and even bacterial agents), as well as numerous immune system abnormalities. This hypothesis focuses on the location of an infection, along the sensory (afferent) of the vagus nerve. Hence CFS is explained by the sensory vagus nerve.

\section{In the Normal State}

The vagus nerve signals the body to rest when it detects a peripheral infection, this signal of fatigue is pathologically exaggerated when an infection is localized on the vagus nerve itself. More specifically, immune cells, including neuro-immune cells called glial cells, The vagus nerve detects infection and initiates the same basic neuro-excitatory response regardless of the type of 
infection. When the glial cells that surround the sensitive vagus nerve are activated by any virus or bacterial infection, their neuroexciting secretions intensify the signaling of the afferent vagus nerve, which is misinterpreted by the brain as evidence of a serious peripheral infection. The brain then initiates disease behavior, which includes fatigue and many other symptoms of CFS than it does:

-Tired

-Insomnia

-Fever

-Loss of appetite

-Musculoskeletal pain (myalgia)

-Hyperalgesia

-Cognitive impairments

-Depression / malaise

-Zinc depletion

-Loss of smell

\section{Relationship Between Vagus Nerve and Coronavirus (Covid-19)}

\section{The coronavirus is a neurotropic virus}

However, neurotropic viruses are the type of pathogen most often associated with CFS.

Because covid-19 (coronavirus) is based on infection of the nervous tissue, this is probably not a coincidence:

This virus is characterized by its affinity to invade neural tissue. The afferent vagus nerve and associated glial cells are important targets for neurotropic virus infection and general immune responses. Neurotropic viruses are characterized by their ability to become latent, particularly in the lymph nodes of nervous and lymphoid tissues. Although the initial infection may have occurred during the first 10 years of life, neurotropic viruses can be reactivated even in healthier adults. Since these viruses tend to remain dormant until reactivation during stress or illness, it follows that CFS patients usually report that their symptoms started during a period of stress or with a normal cold or flu.

While latency tends to occur in nerve tissue, upon reactivation, viral infection spreads to the extracellular space. There, the satellite glial cells envelop the viral particles. These satellite glial cells proliferate and activate, releasing neuro-excitatory mediators such as immune proteins called pro-inflammatory cytokines. The version of pro-inflammatory cytokines is a general 
response from glia and other immune cells such as cells. producing interleukin (white blood cells) to meet any virus or bacteria. These locally released cytokines are detected by the nearest sensory vagus nerve chemoreceptors, causing a signal to the brain. The brain then initiates fatigue and several other symptoms that overlap with CFS. The premise of CFS is that when a neurotropic virus or any other pathogen infects the vagus nerve itself, cytokines are released directly to receptors in the sensitive vagus nerve and this immune system becomes pathologically intense.

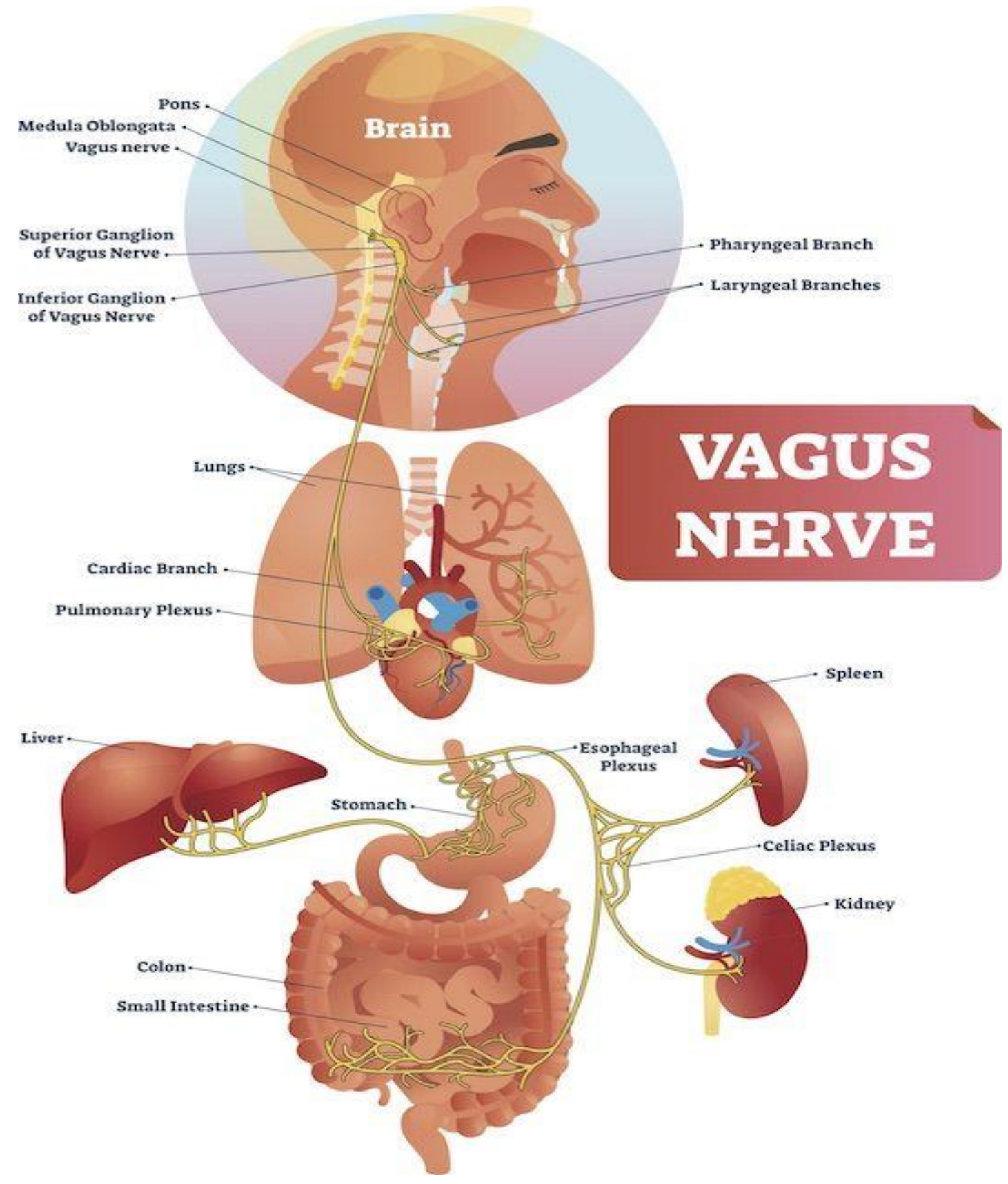

Figure 1. Diagram of the vagus nerve 


\section{The Direct Neurological Signs of The Coronavirus}

Loss of smell and taste, headache, loss of consciousness and cerebrovascular disorders in $80 \%$ of cases. These symptoms do not occur in all patients infected with Covid-19.

\section{Indirect Neurological Signs of The Coronavirus}

Headache (8\%), myalgia (11\%), or even, mental confusion (9\%), fever, respiratory disorder and cognitive fragility in the affected population.

\section{Conclusion}

Chronic Fatigue Syndrome (CFS) is a specific mechanism for explaining the symptoms of covid-19, which should be used as a basis for treatment strategies. The vagus nerve is the conductor of all immune defense mechanisms.

The basic use of biomedical imaging research for the location of an active viral infection such as covid-19 along the vagal pathway from the peripheral to the central nervous system would be of great importance in determining effective treatment.

Functional studies of the vagus nerve should compare highly symptomatic patients with healthy controls. 